\title{
Penile Pain, CTCAE 5.0
}

National Cancer Institute

\section{Source}

National Cancer Institute. Penile Pain, CT CAE 5.0. NCI Thesaurus. Code C146680.

A disorder characterized by a sensation of marked discomfort in the penis. 